\title{
Acute myocardial infarction caused by a septic coronary embolism diagnosed and treated with a thrombectomy catheter
}

\author{
M Taniike, M Nishino, Y Egami, I Kondo, R Shutta, K Tanaka, T Adachi, J Tanouchi, Y Yamada, \\ K Kawano
}

Acute myocardial infarctions are common in bacteraemia but are seldom diagnosed during life. A 64 year old man with severe chest pain who had fever for several days due to possible bacteraemia was shown by ECG and echocardiography to have possible lateral infarction. Immediate coronary angiography showed possible thrombus in the left circumflex artery, which was treated by thrombectomy catheter. Bacterial thrombus was removed and was verified by histological examination. A stent was implanted without complications. Acute myocardial infarction caused by septic embolism is usually fatal; however, thrombectomy may be useful in these cases.

$\mathrm{P}$ erera et $a l^{1}$ described a fatal case of acute myocardial infarction that arose from embolism of bacterial vegetation. Thromboembolic complications including myocardial infarction are common in patients with bacteraemia with or without endocarditis, but acute myocardial infarctions are seldom diagnosed during life. ${ }^{2}$ Recently, thrombectomy catheters have been used to treat acute myocardial infarctions in clinical settings. ${ }^{3}$ We report a case of acute myocardial infarction caused by bacterial embolism in a major epicardial artery, diagnosed and treated with a RESCUE thrombectomy catheter (Scimed/BSC, Natick, Massachusetts, USA).

A 64 year old man with chest pain was referred to the Division of Cardiology from the Division of Nephrology in our hospital. He had been receiving haemodialysis for 10 years and one month previously had undergone femoral-popliteal artery bypass surgery because of arteriosclerosis obliterans. After surgery, he remained in the Division of Nephrology for haemodialysis and rehabilitation. Owing to occlusion of the dialysis shunt, a transient dialysis catheter was inserted into his jugular vein one week before the onset of chest pain. He consequently developed a fever and thus the catheter was removed three days later. On the day of severe chest pain (two days after removal of the catheter) he was febrile, an ECG showed ST segment depression in leads I, II, III, aVF, and $V_{3}$ to $V_{6}$, and echocardiography showed lateral wall motion abnormality but no findings indicating infective endocarditis. Urgent coronary angiography showed almost abrupt occlusion of the proximal left circumflex coronary artery caused by possible thrombus (fig lA). We immediately performed coronary aspiration with a RESCUE thrombectomy catheter (fig 1B) and as a result a large amount of material was collected. After aspiration, we performed balloon angioplasty and implanted a stent, which achieved successful reperfusion (TIMI (thrombolysis in myocardial infarction) flow grade $3^{4}$ ). The maximum creatine kinase concentration was $880 \mathrm{IU} / \mathrm{l}$ and the patient was treated with antibiotics and anticoagulants.

The material collected by the RESCUE catheter measured $2 \mathrm{~mm}$. Histological findings were Gram positive streptococcus in a fibrin thrombus (fig 2). We feared that implanting the stent without aspiration might have caused a mycotic coronary aneurysm at the location of stent implantation. ${ }^{56}$ The mechanism of septic coronary embolism in this case was not clear. We speculated that a small vegetation on the valve, undetected by transthoracic echocardiography, might have
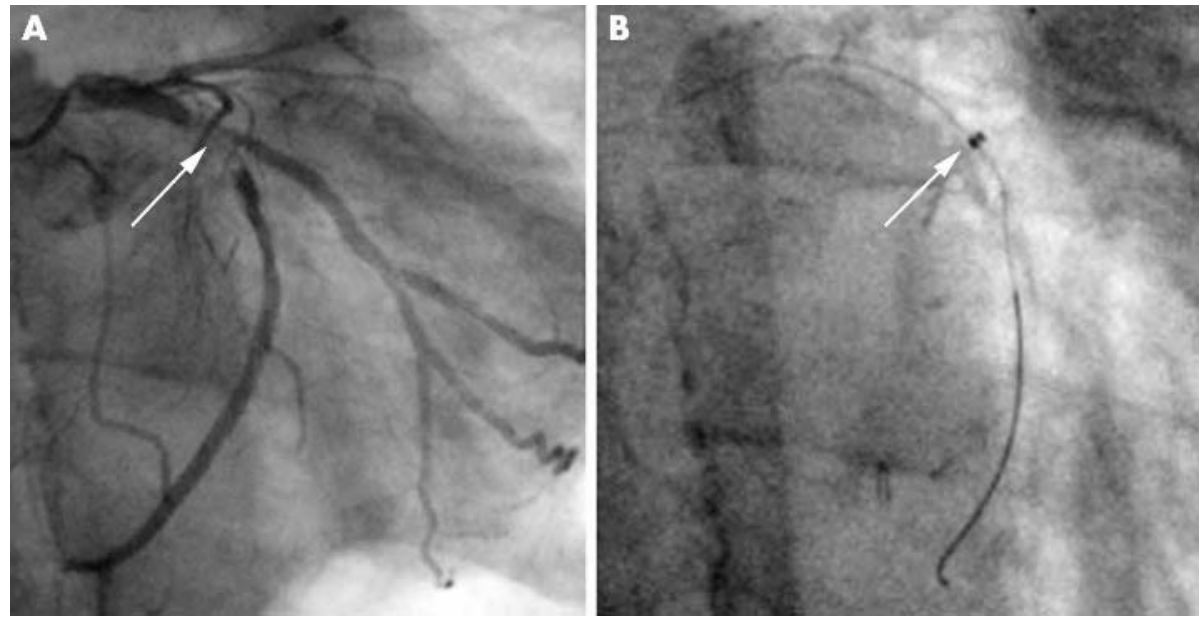

Figure 1 (A) Coronary angiogram showing a possible thrombus (contrast defect, arrow) in the proximal left circumflex coronary artery. (B) Coronary aspiration with a RESCUE thrombectomy catheter. The arrow shows the tip of the catheter located in the thrombus. 


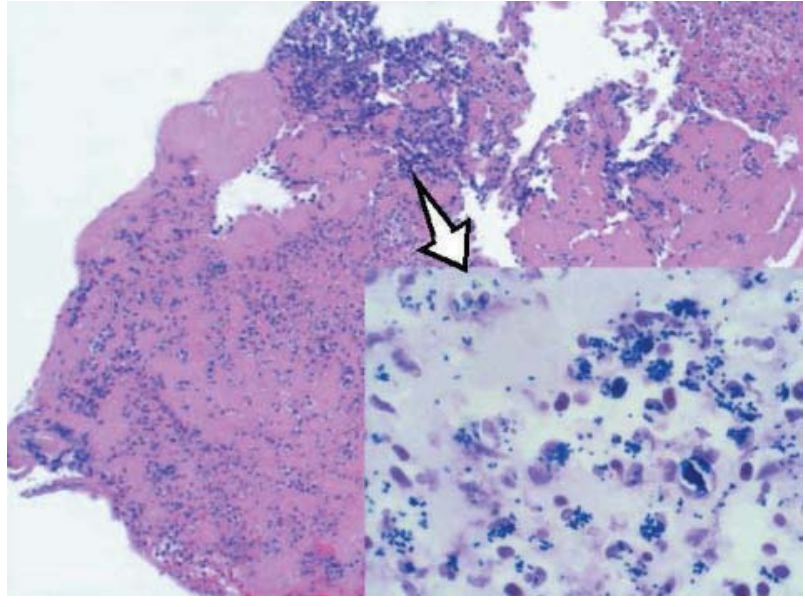

Figure 2 Histological findings of the material retrieved with a RESCUE thrombectomy catheter (haematoxylin and eosin stain, original magnification $\times 20$ ). Gram's staining (inset) showing numerous Gram positive streptococci in a fibrin thrombus that was coincident with bacterial embolism (original magnification $\times 200$ ).

caused embolisation of the coronary artery or enhanced thrombogenic state due to sepsis resulting in production of bacterial thrombi. Acute myocardial infarction caused by septic embolism is usually fatal; however, thrombectomy may be useful in these cases.

\section{Authors' affiliations}

M Taniike, M Nishino, Y Egami, I Kondo, R Shutta, K Tanaka, T Adachi, J Tanouchi, Y Yamada, K Kawano, Division of Cardiology and Clinical Pathology, Osaka Rosai Hospital, Osaka, Japan

Correspondence to: Dr Masami Nishino, Division of Cardiology, Osaka Rosai Hospital, 1179-3, Nagasone-cho, Sakai-city, Osaka 591-8025, Japan; mnishino@orh.go.jp

Accepted 3 November 2004

\section{REFERENCES}

1 Perera R, Noack S, Dong W. Acute myocardial infarction due to septic coronary embolism. N Engl J Med 2000;342:977-8.

2 Valtonen V, Kuikka A, Syrianen J. Thrombo-embolic complications in bacteraemic infections. Eur Heart J 1993;14:20-3.

3 Kotani J, Nanto S, Mintz GS, et al. Plaque gruel of atheromatous coronary lesion may contribute to the no-reflow phenomenon in patients with acute coronary syndrome. Circulation 2002;106:1672-7.

4 The TIMI Study Group. The thrombolysis in myocardial infarction (TIMI) trial. N Engl J Med 1985;312:932-6.

5 Matsumoto $M$, Konishi Y, Miwa S, et al. Mycotic aneurysm of the left coronary artery. Ann Thorac Surg 1998;65:841-2.

6 Osevala MA, Heleotis TL, DeJene BA. Successful treatment of a ruptured mycotic coronary artery aneurysm. Ann Thorac Surg 1999;67:1780-2. 